\title{
Pharmacologically controlled drinking in the treatment of alcohol dependence or alcohol use disorders: a systematic review with direct and network meta- analyses on nalmefene, naltrexone, acamprosate, baclofen and topiramate
}

\author{
Clément Palpacuer', Renan Duprez², Alexandre Huneau', Clara Locher ${ }^{1,3,4}$, Rémy Boussageon ${ }^{5}$, \\ Bruno Laviolle $^{1,3,4}$ \& Florian Naudet ${ }^{1,6}$
}

Inserm, CIC I 14 Clinical Investigation Centre, Rennes, France,' Fondation Saint Jean de Dieu, Centre Hospitalier Dinan/St Brieuc, Dinan, France, ${ }^{2}$ Rennes University Hospital, Department of Biological and Clinical Pharmacology and Pharmacovigilance, Rennes, France, ${ }^{3}$ Rennes I University, Laboratory of Experimental and Clinical Pharmacology, Rennes, France, ${ }^{4}$ Département de Médecine Générale, Faculté de Médecine et de Pharmacie, Université de Poitiers, Poitiers, France ${ }^{5}$ and MetaResearch Innovation Center at Stanford (METRICS), Stanford University, Palo Alto, CA, USA

\begin{abstract}
Background and Aims Pharmacologically controlled drinking in the treatment of alcohol dependence or alcohol use disorders (AUDs) is an emerging concept. Our objective was to explore the comparative effectiveness of drugs used in this indication. Design Systematic review with direct and network meta-analysis of double-blind randomized controlled trials (RCTs) assessing the efficacy of nalmefene, naltrexone, acamprosate, baclofen or topiramate in non-abstinent adults diagnosed with alcohol dependence or AUDs. Two independent reviewers selected published and unpublished studies on Medline, the Cochrane Library, Embase, ClinicalTrials.gov, contacted pharmaceutical companies, the European Medicines Agency and the Food and Drug Administration, and extracted data. Setting Thirty-two RCTs. Participants A total of 6036 patients. Measurements The primary outcome was total alcohol consumption (TAC). Other consumption outcomes and health outcomes were considered as secondary outcomes. Findings No study provided direct comparisons between drugs. A risk of incomplete outcome data was identified in 26 studies (81\%) and risk of selective outcome reporting in 17 (53\%). Nalmefene [standardized mean difference $(\mathrm{SMD})=-0.19,95 \%$ confidence interval $(\mathrm{CI})=-0.29$, $-0.10 ; I^{2}=0 \%$, baclofen $(\mathrm{SMD}=-1.00,95 \% \mathrm{CI}=-1.80,-0.19$; one study) and topiramate $(\mathrm{SMD}=-0.77,95 \%$ $\left.\mathrm{CI}=-1.12,-0.42 ; I^{2}=0 \%\right)$ showed superiority over placebo on TAC. No efficacy was observed for naltrexone or acamprosate. Similar results were observed for other consumption outcomes, except for baclofen (the favourable outcome on TAC was not reproduced). The number of withdrawals for safety reasons increased under nalmefene and naltrexone. No treatment demonstrated any harm reduction (no study was powered to explore health outcomes). Indirect comparisons suggested that topiramate was superior to nalmefene, naltrexone and acamprosate on consumption outcomes, but its safety profile is known to be poor. Conclusions There is currently no high-grade evidence for pharmacological treatment to control drinking using nalmefene, naltrexone, acamprosate, baclofen or topiramate in patients with alcohol dependence or alcohol use disorder. Some treatments show low to medium efficacy in reducing drinking across a range of studies with a high risk of bias. None demonstrates any benefit on health outcomes.
\end{abstract}

Keywords Alcohol dependence, alcohol use disorders, controlled drinking, meta-analysis, pharmacotherapy, randomized controlled trial.

Correspondence to: Clément Palpacuer, Centre d'Investigation Clinique INSERM 1414, Hôpital de Pontchaillou, 2 rue Henri le Guilloux, 35033 Rennes cedex 9 , France. E-mail: clement.palpacuer@gmail.com

Submitted 18 March 2017; initial review completed 16 May 2017; final version accepted 28 July 2017

\section{INTRODUCTION}

Pharmacologically controlled drinking in the treatment of patients suffering from alcohol dependence or alcohol use disorders (AUDs) is an emerging concept [1,2]. In Europe, nalmefene, an opioid antagonist that acts on the urge to consume alcohol, was the first treatment approved by the European Medicines Agency (EMA) for this indication [3]. 
Nevertheless, the evidence for its approval was contested [4,5]. As no difference was observed on health outcomes, which is the aim of a harm reduction strategy, the approval was based on small differences versus placebo on alcohol consumption outcomes, only observed in a subgroup defined a posteriori in studies with a possible attrition bias (nalmefene tolerance was poor) [6-8]. In addition, nalmefene has not been compared with other active treatments, in particular naltrexone [9], another opioid antagonist that has previous approval in maintaining abstinence and that has already been used off-label in reducing consumption, with some evidence suggesting a possible interest for this indication [10].

Other drugs acting on the gamma-aminobutyric acid (GABA)ergic system have also been studied for this indication [11]. All have already been used, more or less officially, to help reduce alcohol consumption: (1) baclofen has been used in France under a 'temporary recommendation for use'; (2) acamprosate is the calcium salt of N-acetylhomotaurine and has had approval for post-withdrawal maintenance of alcohol abstinence and (3) topiramate is an anti-epileptic drug that is considered as an emerging alternative.

While widely diffused guidelines $[12,13]$ and major reviews [14] now promote this approach as part of a harm reduction strategy, there is a need to assess the evidence that supports the use of these drugs.

The objective of this study was to test the efficacy of treatments (1) from head-to-head comparisons, and (2) from indirect comparisons using a network metaanalysis.

\section{METHODS}

\section{Design}

A standard meta-analysis protocol was developed and registered before the beginning of the study in the PROSPERO database (systematic review registration-PROSPERO 2015: CRD42015019841). Double-blind randomized controlled trials (RCTs) assessing the efficacy of nalmefene, naltrexone, acamprosate, baclofen or topiramate against each other or against placebo were considered for inclusion in the meta-analysis.

\section{Eligibility criteria}

We reviewed studies involving adults (aged 18 years and over) with a diagnosis of alcohol use disorders (AUDs) and/or alcohol dependence. We focused on non-abstinent patients. As detoxification is generally obtained after 5-7 days, we included patients with fewer than 5 days' abstinence before the beginning of the study [15]. Therefore, studies were not included when longer abstinence (or detoxification) was an explicit inclusion criterion. In addition, studies with patients presenting systematic physical or psychological comorbidity were not included.

Studies were eligible if they focused on the comparison of oral formulations of (1) nalmefene, (2) naltrexone, (3) acamprosate, (4) baclofen or (5) topiramate with each other or with a placebo. Only monotherapies were considered. In case of multiple-dose studies, only the dose closest to the recommended dose was taken into account (see Supporting information, Appendix S1, p. 2). We reviewed RCTs systematically without any limitation in terms of duration. Only study reports in English, French, German and Spanish were considered.

\section{Search strategy and study selection process}

Eligible studies were identified from PubMed/Medline, the Cochrane library and Embase, including congress abstracts. The searches were conducted in April 2015. An update of the search was performed for the Pubmed database in June 2016. The same algorithm was used for all electronic databases: "Nalmefene OR Baclofen OR Acamprosate OR Topiramate OR Naltrexone AND Alcohol' with the filter 'Clinical Trial'. Two reviewers (A.H. and R.D.) reviewed independently the titles and abstracts of all citations identified by the literature search. The full text of relevant studies was examined. All disagreements were resolved by consensus or by consultation with another reviewer (F.N.). To avoid omission of any studies, all the references identified were compared to (1) references included in a previous comprehensive meta-analysis on pharmacotherapy of alcohol use disorders [16] that were shared by the author and (2) references included in a previous indirect comparison of nalmefene and naltrexone [17].

Unpublished studies were also searched for by consulting the ClinicalTrials.gov website and key organizations such as the Food and Drug Administration (FDA) and EMA. Pharmaceutical companies (Novartis, Janssen Cilag, Merck Sereno, Bristol Myers Squibb and Lundbeck) were also contacted to obtain information concerning their studies. When needed, the authors of the abstracts were contacted for further information and were asked for references of the studies. If no response was obtained to a first request, they were contacted a second time. We also contacted authors of recent meta-analyses on the pharmacological management of AUDs and asked them if they would agree to share their data.

\section{Assessment of methodological quality}

Two reviewers (C.P. and R.D.) assessed independently the risks of bias in each study included in the meta-analysis using the Cochrane Collaboration tool for assessing risk of bias [18]. 


\section{Data collection}

Two reviewers (C.P. and R.D.) collected data from the studies included using a data extraction sheet, based on the Cochrane Handbook for Systematic Reviews of Interventions guidelines [19]. Disagreements were first resolved by consensus and then by consulting a third reviewer for arbitration (F.N.). Studies appearing to duplicate authors, treatment comparisons, sample sizes and outcomes were checked one against the other to avoid any duplicates and to avoid integrating data from several reports on the same study. For each study included, information was extracted on (1) characteristics of the study [year, country, co-treatments (psychological interventions and their type), number of arms, funding], (2) characteristics of trial participants (age, gender, number of patients included in the analysis, population analysed), (3) type of intervention (treatments and comparators, duration) and (4) outcome measures as detailed below.

\section{Outcome measures}

Our primary outcome was total alcohol consumption (TAC). The choice of TAC was based on our previous meta-analysis on nalmefene [8], and on the fact that the rationale for using pharmacologically controlled drinking stems from the idea that if individuals reduce their total alcohol consumption, they reduce their levels of risk accordingly. Other consumption outcomes were considered as secondary outcomes, namely: (1) the number of heavy drinking days (HDD), defined as a day with a high consumption level, (2) the number of non-drinking days, (3) the number of drinking days and (4) the number of drinks per drinking day. We also extracted safety outcome data: (1) adverse events, (2) serious adverse events, (3) withdrawals from the study, (4) withdrawals for safety reasons and (5) mortality.

\section{Data analysis}

For consumption outcomes, the treatment effects were expressed as standardized mean differences (SMD, Hedge's $g$ ) between arms, because these outcomes were likely to be assessed in different ways in the different studies. The efficacy index used for safety outcomes was the odds ratio (OR). All outcomes were presented with their 95\% confidence interval (CI). The analytical plan was divided into two main steps: we first carried out conventional meta-analyses focusing on direct, pair-wise comparisons. We then performed a network meta-analysis of all studies included to estimate the effects of each active treatment as compared to all the others. All analyses were performed using R statistical software [20] and the meta [21], netmeta [22] and metafor [23] libraries. Results were presented according to PRISMA (Preferred Reporting Items for Systematic Reviews and Meta-Analyses) format [24] and its extension for network meta-analyses [25].

\section{Direct comparisons}

An estimate of the overall effect (summary measure) was calculated using a random-effects model (DerSimonian \& Laird method). For mortality, serious adverse events and withdrawals for safety reasons, which are rare outcomes, we used Peto's OR method [26], as recommended by the Cochrane Collaboration for handling sparse data [19]. Heterogeneity was evaluated using a visual inspection of the forest plot, the $Q$ statistic and the $I$-squared $\left(I^{2}\right)$ index.

\section{Indirect comparisons}

Network meta-analyses enabling indirect comparisons were performed using the graph-theoretical method [27], a frequentist approach. We used a design-based breakdown of Cochrane $Q$ and the $I^{2}$ index for assessing the heterogeneity in the whole network, the homogeneity within designs and, if applicable, the homogeneity/consistency between designs. A random-effects model was used for all indirect comparisons.

In addition to the estimation of summary measures and their 95\% CI, we estimated the P-scores enabling a ranking of all treatments which mostly follow that of the point estimates, but take precision into account. These P-scores measure the mean extent of certainty that a treatment is better than the competing treatments [28].

\section{Risk of bias across studies}

Publication bias was investigated graphically using funnel plots for each direct meta-analysis of our principal outcome when there were at least four studies. Funnel plot asymmetry was tested using the rank correlation test when there were at least 10 studies [29].

\section{Minor changes to the initial protocol}

While we had planned initially to focus on consumption outcomes, we added safety outcomes before data extraction because (1) these outcomes are necessary to estimate the risk/benefit ratio of the drugs considered and (2) a reduction of mortality and/or in serious adverse events are expected benefits of a harm reduction approach.

We assessed the quality of evidence contributing to each network estimate for total alcohol consumption (TAC) using the Grading of Recommendations Assessment, Development and Evaluation (GRADE) framework [30]. As it appeared that data on HDDs were more complete than for TAC, we also evaluated the quality of the evidence with the GRADE method for this outcome. 
When substantial heterogeneity $\left(I^{2}>50 \%\right)$ was found in direct comparisons, meta-regressions were performed, a posteriori, to explore whether the year of study, the definition of non-abstinence (no eligibility criteria requiring a period of abstinence before inclusion versus presence of a criterion specifying four or fewer abstinent days before inclusion) or the sex ratio (studies including men only versus other studies) were possible effect modifiers. When effect modifiers were suspected, an analysis excluding studies that might have introduced heterogeneity was performed as a post-hoc sensitivity analysis.

\section{Role of the funding source}

The funder of the study had no role in study design, data collection, data analysis, data interpretation or writing of the report. The corresponding author had full access to all the data in the study and had final responsibility for the decision to submit for publication.

\section{RESULTS}

A flow-chart detailing the study selection process is given in Fig. 1. After adjusting for duplicates, the searches provided a total of 2680 citations. Of these, 158 studies were assessed for eligibility. Thirty-two RCTs involving 6036 patients met our eligibility criteria and were included in the analysis [31-60]. The EMA provided us with access to all study reports concerning nalmefene (including two unpublished eligible studies), because the drug had received approval in Europe. We did not have access to study reports either from the EMA for other drugs or from the FDA for all drugs. For one study [54], the main analysis was presented as part of a systematic review [61].

\section{Study characteristics}

All 32 RCTs included in the meta-analysis were placebocontrolled. They were published between 1994 and



Figure I Flow diagram 
2015. Their main characteristics are summarized in Table 1. The studies compared the effects of oral nalmefene $(n=9)$, naltrexone $(n=14)$, acamprosate $(n=1)$, baclofen $(n=4)$ or topimarate $(n=4)$ against placebo. No study provided direct comparisons between active treatments. Placebo was the most frequently studied intervention, with 2780 patients. Nalmefene and naltrexone were the most widely studied drugs with, respectively, 1693 and 850 patients receiving one of the two medications in the trials. The other drugs were studied less frequently with, respectively, 258, 106 and 349 patients for acamprosate, baclofen and topiramate. Generally, study durations were longer for nalmefene studies [median: 24 weeks, interquartile range $(\mathrm{IQR})=12-28]$ than for naltrexone (median: 12 weeks, IQR = 10-16), baclofen (median: 12 weeks, IQR $=4-12$ ) and topiramate (median: 12 weeks, IQR $=12-$ 12). The study duration of the acamprosate trial was 24 weeks. There were no major differences across studies in terms of subject age (except for one naltrexone study where the adults included were younger than in the others [49]) and sex ratio (except for three naltrexone studies conducted in men only $[38,47,48])$. Except for one nalmefene trial (CPH-101-0701) and one naltrexone trial [51], all patients received a psychological co-intervention (including medical management programmes) during the study periods.

\section{Risk of bias within studies}

The risk of bias assessment is reported graphically in Fig. 2 . Twenty-six studies (81\%) were classified as having an unclear or a high risk of incomplete outcome data due to the large number of withdrawals. Extractable data were based on a complete-case population for 26 trials (81\%). Therefore, an attrition bias cannot be excluded. Seventeen studies (53\%) were considered to present an unclear or a high risk of selective outcome reporting, as they did not mention a protocol registration number that allowed us to check whether all outcomes were reported. Because we had access to study reports, we were more confident in the exhaustiveness of the nalmefene studies in comparison with other drugs. All these studies lacked consensus on the ways of measuring and/or reporting outcomes, as detailed in the Supporting information, Appendix S1 (p. 4). Therefore, the analyses of the different outcomes were based on a different number of studies and participants.

\section{Data analysis}

Direct comparisons

For all outcomes, the results of direct analyses (number of studies, efficacy indices, $I^{2}$ index) are summarized in Table 2 and presented graphically with individual study results in the Supporting information, Appendix S1 (p. 8). In general, substantial heterogeneity was shown for the naltrexone and topiramate studies. In particular, for naltrexone, while some studies were in favour of a superiority of the drug, one study found a significant superiority of placebo [41].

Regarding the primary outcome (TAC), it was reported in only seven (78\%) nalmefene studies, five (36\%) naltrexone studies, one $(100 \%)$ acamprosate study, one $(25 \%)$ baclofen study and two (50\%) topiramate studies. Nalmefene, topiramate and baclofen showed a greater decrease in TAC than placebo. Data on HDD were more complete. The same results were observed, except for baclofen, which did not show any superiority over placebo. Compared to placebo, topiramate increased significantly the number of non-drinking days and reduced the number of drinking days. Nalmefene reduced significantly the number of drinks per drinking days. For nalmefene, estimated effect sizes were always small. Conversely, the effect size for baclofen was large on TAC but inconsistent on other criteria. Topiramate consistently showed medium to large effect sizes on all consumption outcomes.

No difference was found for any drug on mortality and serious adverse events. More adverse events and withdrawals for safety reason were evidenced for naltrexone and nalmefene, and more withdrawals for nalmefene.

\section{Indirect comparisons}

For all outcomes, evidence of differences between drugs was derived from indirect comparisons, as the networks were star-shaped. Heterogeneity ranged from minor to substantial (range of $I^{2}=0-61.5, I^{2}$ greater than 50\% found for three outcomes). The consistency hypothesis could not be ascertained because there was no direct comparison with active treatments. For TAC (Table 3), based on the evidence detailed for direct comparisons, topiramate was superior to nalmefene, naltrexone and acamprosate. Similarly, baclofen showed superiority over naltrexone and acamprosate on TAC. There was no evidence for a difference between nalmefene and naltrexone. The superiority of topiramate seemed to be consistent on all the other consumption outcomes while the superiority of baclofen was not. No significant difference was found across drugs on the safety outcomes (see Supporting information, Appendix S1, p. 62), except for withdrawals for safety reasons, as presented in Table 3. For all outcomes, detailed contrast tables are provided in the Supporting information, Appendix $S 1$ (p. 62). Network geometry is presented in Fig. 3 for TAC and the withdrawals for safety reasons, and in the Supporting information, Appendix S1 (p. 59) for the other outcomes.

Results are summarized visually with the ranking according to P-scores in Fig. 4. Overall, the higher the P-scores for the consumption outcomes, the lower the 


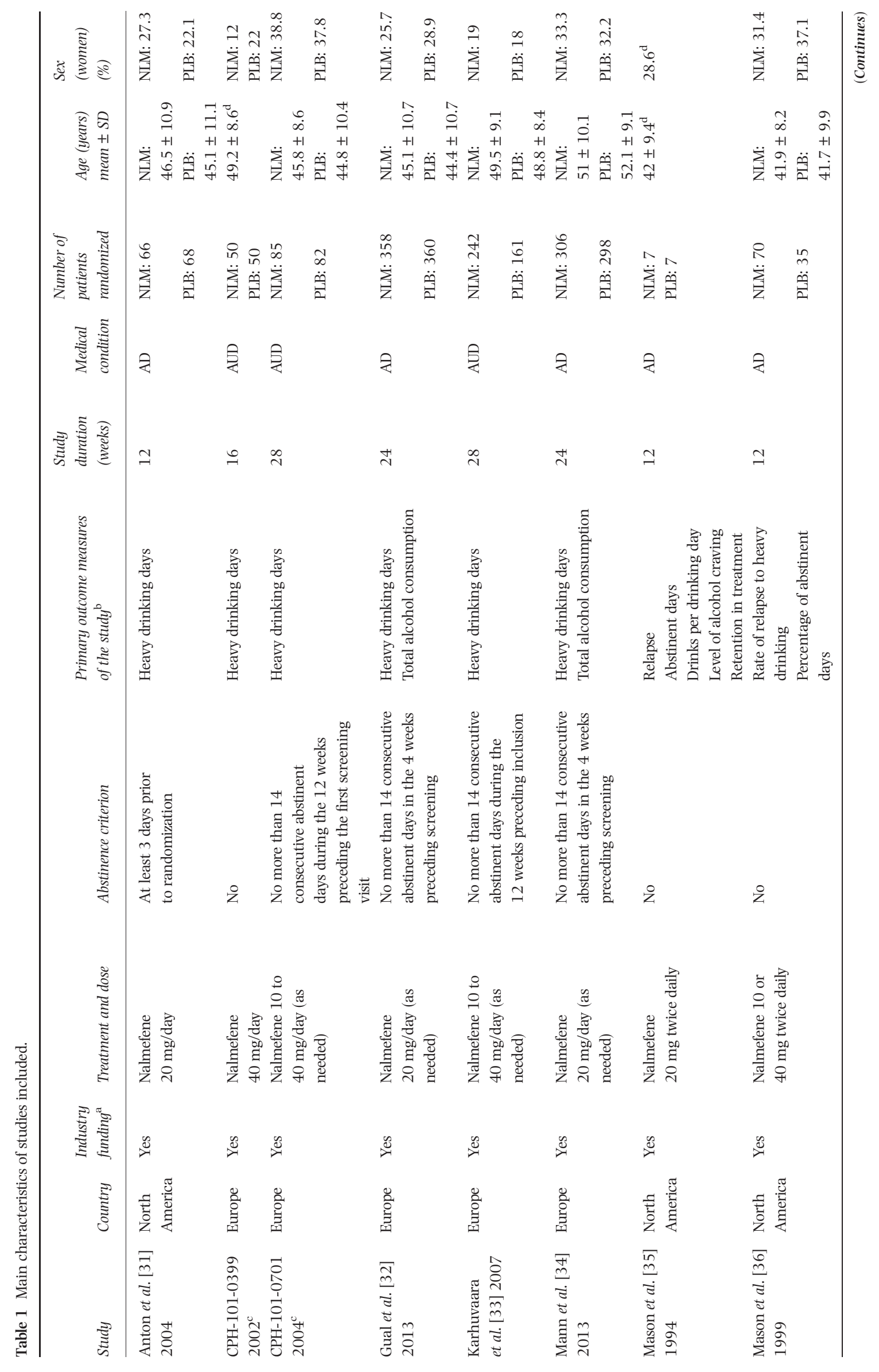




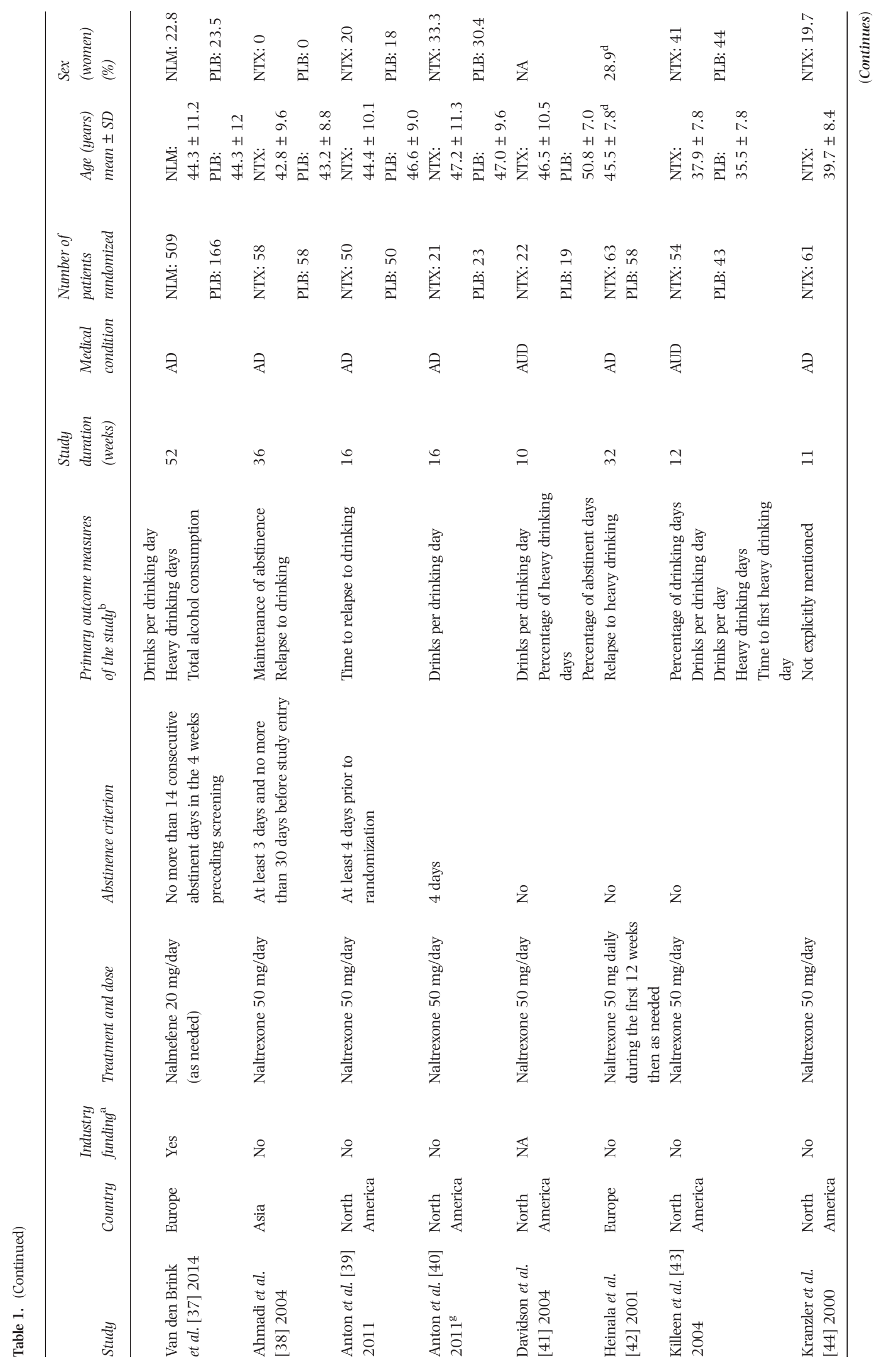




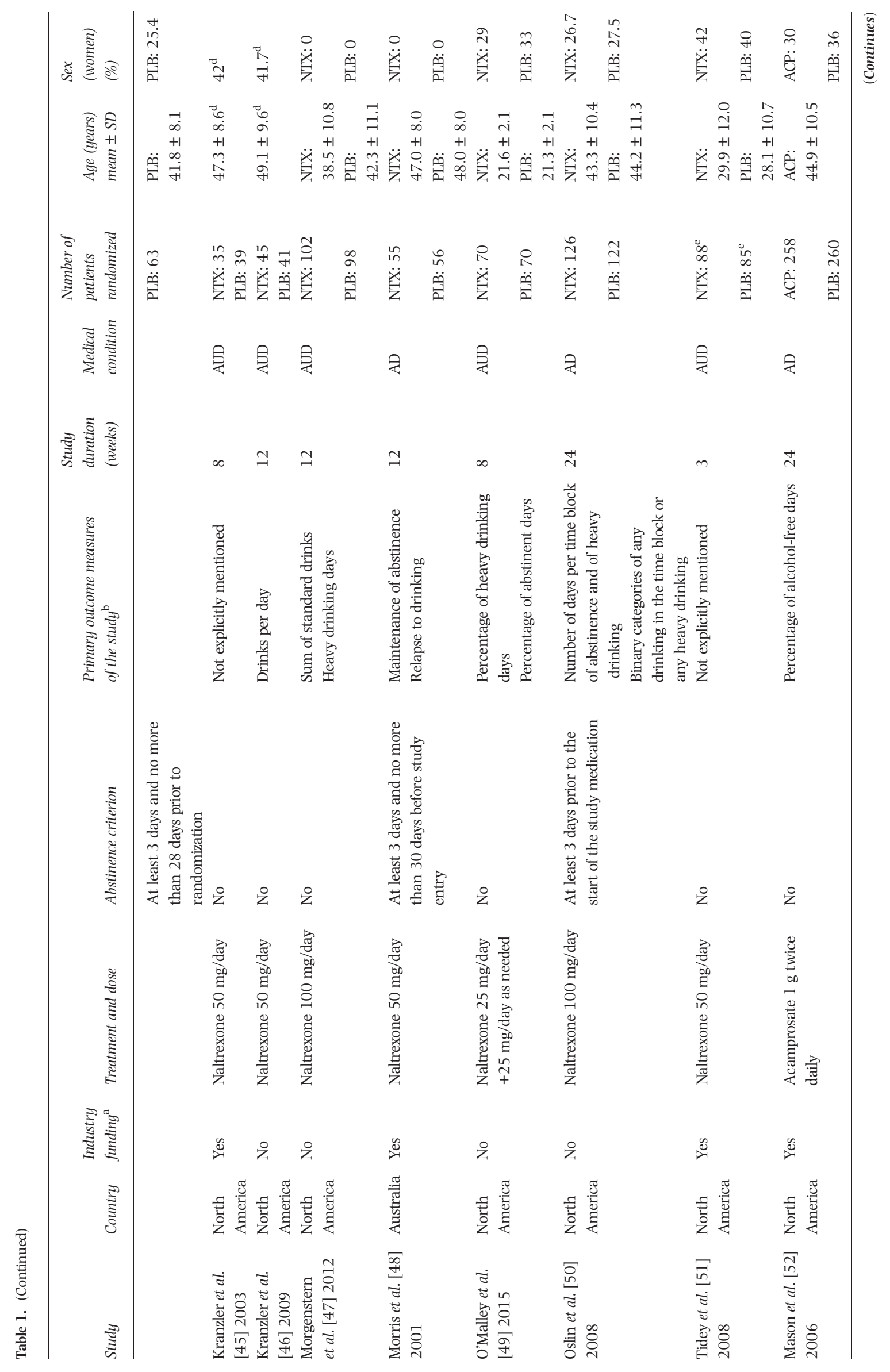







P-scores for the safety outcomes. We also noticed that the safety profiles of nalmefene and naltrexone were similar.

Risk of publication bias and selective outcome reporting

We only investigated the existence of a publication bias for our principal outcome. When applicable, funnel plots are presented in the Supporting information, Appendix S1 (p. 8). In general, the small number of studies per outcome prevented any meaningful use of this strategy. Therefore, we consider that small study effects cannot be ruled out. For example, concerning topiramate on the outcome HDD, it is noticeable that the largest study against placebo in terms of numbers of patients included found the smallest effect size [standardized mean difference $(\mathrm{SMD})=-0.2$ ]. In addition, as stated in the risk assessment paragraph, we estimated that selective outcome reporting was possible for the studies included.

\section{Investigation of heterogeneity}

We performed meta-regression analyses for outcomes associated with substantial heterogeneity $\left(I^{2}>50 \%\right)$. The definition of abstinence and the year of study were not identified as possible effect modifiers. We identified the sex ratio as a possible effect modifier, as it was associated significantly with the number of drinks per drinking day for naltrexone studies. A sensitivity analysis was performed after having removed studies including only men. Overall, results were not changed, and some heterogeneity was still unexplained.

\section{Quality of evidence contributing to each network estimate}

According to the GRADE method, the quality of evidence was very low for the overall ranking of treatments for TAC and HDD (see Supporting information, Appendix S1, p. 66).

\section{DISCUSSION}

\section{Statement of principle findings}

Regarding our primary outcome (TAC), nalmefene, baclofen and topiramate showed superiority over placebo. Furthermore, indirect comparisons showed superiority of topiramate over nalmefene, naltrexone and acamprosate. It is noteworthy that the reporting of TAC (our primary end-point) was less consistent than the reporting of other consumption outcomes such as HDD. However, similar trends were observed for the other consumption outcomes, except in the case of baclofen (the positive results for baclofen on TAC were based on a single small study). For all treatments except topiramate, effect sizes were small or inconsistent. Furthermore, nalmefene and naltrexone were associated with a significant increase in withdrawals from 


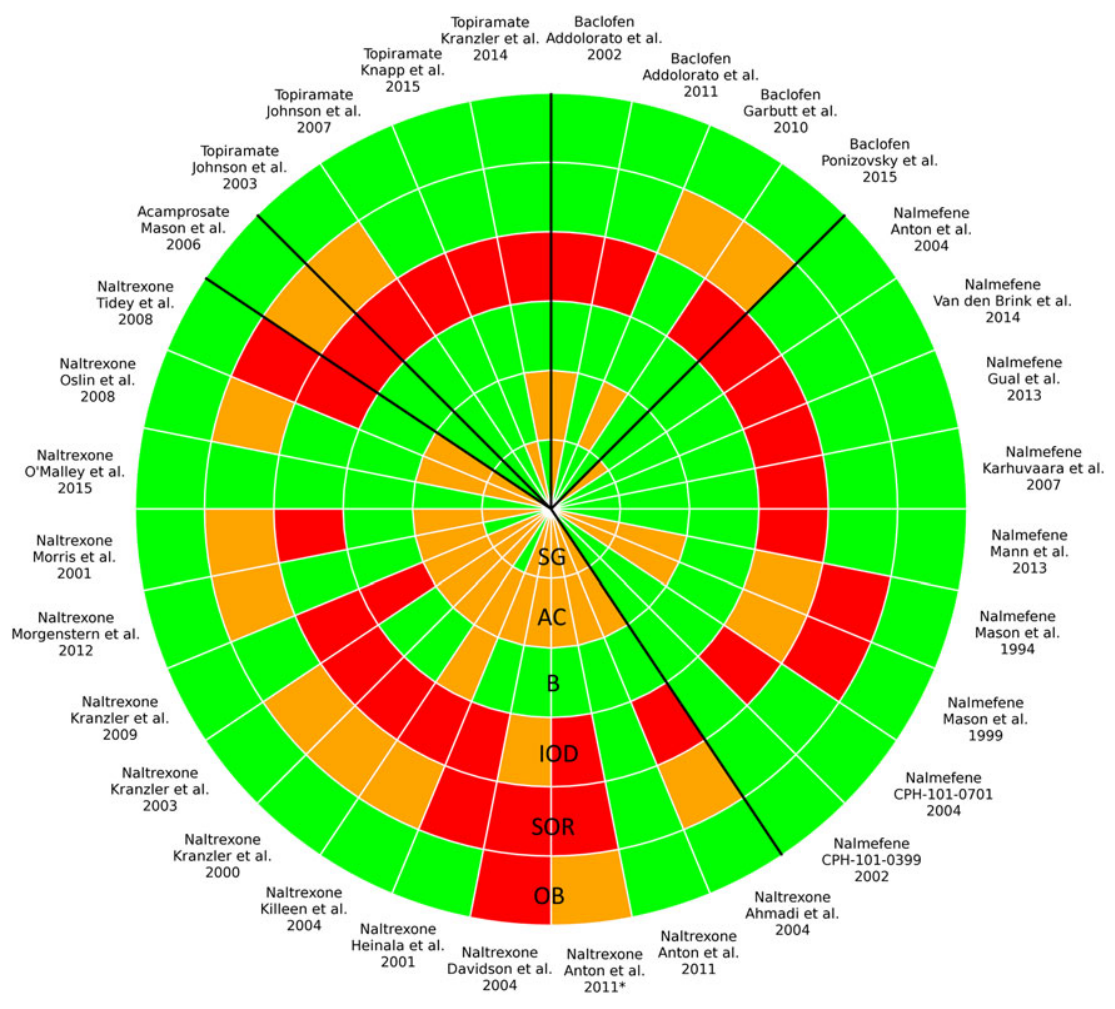

Risk of bias $\quad$ HR UR LR

Figure 2 Quality evaluation of included studies according to the Cochrane Collaboration tool for assessing risk of bias. SG = sequence generation; $\mathrm{AC}=$ allocation concealment; $\mathrm{B}=$ blinding; $\mathrm{IOD}$ = incomplete outcome data; $\mathrm{SOR}=$ selective outcome reporting; $\mathrm{OB}=$ other bias. For each item, the risk of bias was classified as 'low risk' ( $L R$ ), 'high risk' (HR) or 'unclear risk' (UR), with the last category indicating either lack of information or uncertainty over the potential for bias.

the study and withdrawals for safety reasons, which raises concerns about a plausible attrition bias.

Overall, there is no evidence for a significant reduction in serious adverse events or in mortality. As for studies on substances aiming to maintain abstinence [16], no study was designed with sufficient power, and study durations were inadequate to investigate these health outcomes. In addition, any pharmacological approach that might benefit patients by reducing their alcohol consumption might also harm them because of possible safety issues. Therefore, if we consider the reduction of consumption as a valid surrogate outcome, it is not certain that its validity for a given drug can be transposed to another drug with a distinct safety profile. For these reasons, we have advocated longterm mega-trials exploring health outcomes (including large, randomized, controlled cluster trials) in targeted populations [7]. One could argue that adequately designed trials on health outcomes would be unfeasible (too large and too long) and suggest that this evidence could be obtained from observational studies on large administrative databases. This is even more challenging. In our opinion, non-randomized observational evidence of this kind might not be robust enough to resist the major conflicts of interest inherent in the evaluation of pharmaceuticals. Weaknesses of these study designs include indication bias (especially when comparing drugs with absence of treatment) and non-measured confounders. Without entering further into this debate, there is a great need for consensus to move the field forward. Otherwise, we will continue to produce lowquality evidence that maintains the unacceptable status quo, as illustrated by our meta-analysis.

\section{Strengths and weaknesses of the study}

Regarding the strengths and weaknesses of the study, one major strength of network meta-analyses is that they provide a broad picture of the evidence for specific conditions, and make it possible understand how much evidence exists for each treatment [62]. In this case, the network metaanalysis enabled us to appraise critically the literature on treatments used commonly to reduce alcohol consumption in alcohol dependence and AUDs. It showed how the emerging concept of pharmacologically controlled drinking is based currently on results from studies with a high risk of bias (including attrition bias due to the high rates of withdrawal). Pinpointing these shortcomings is a crucial result 


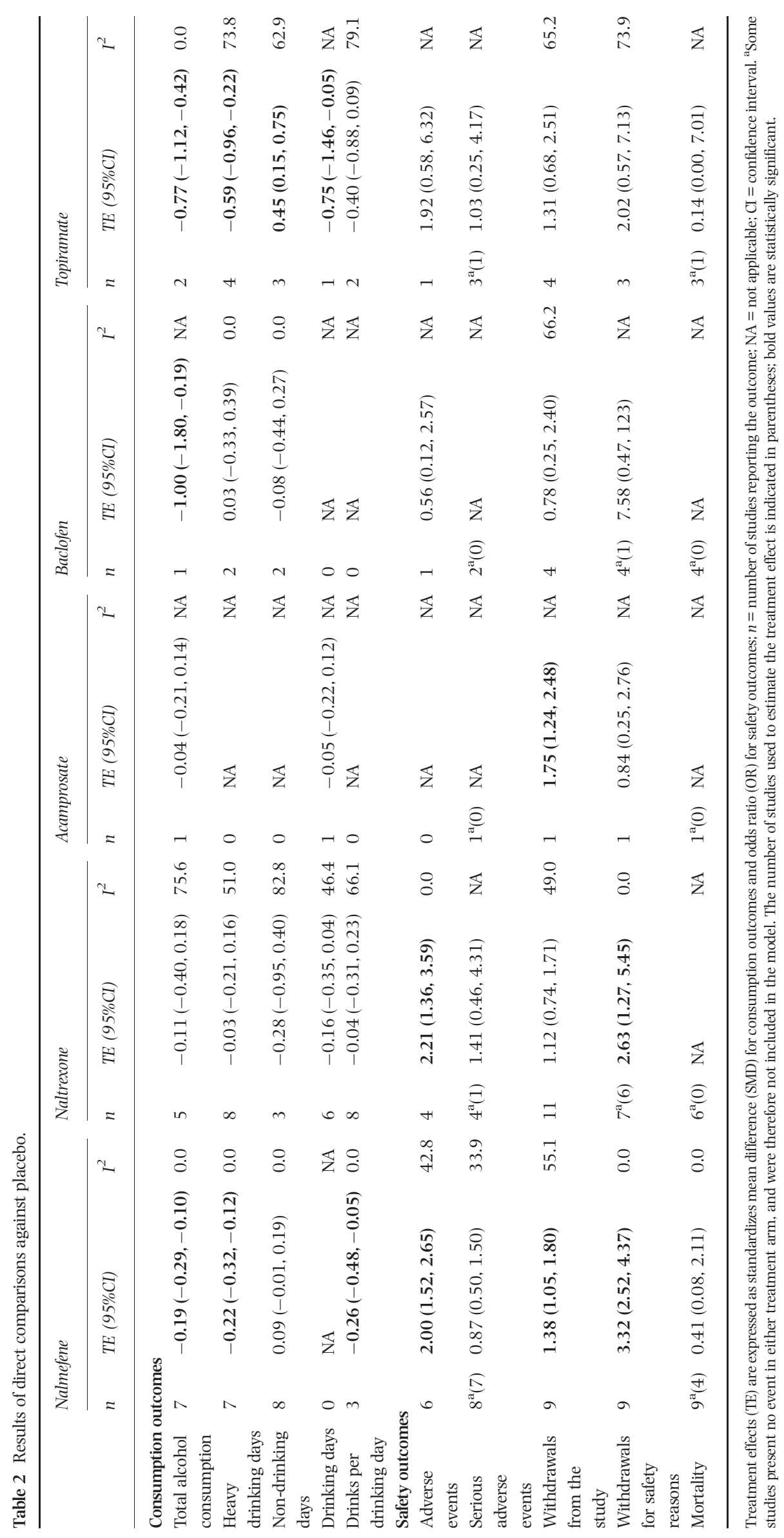


Table 3 Results of indirect comparisons.

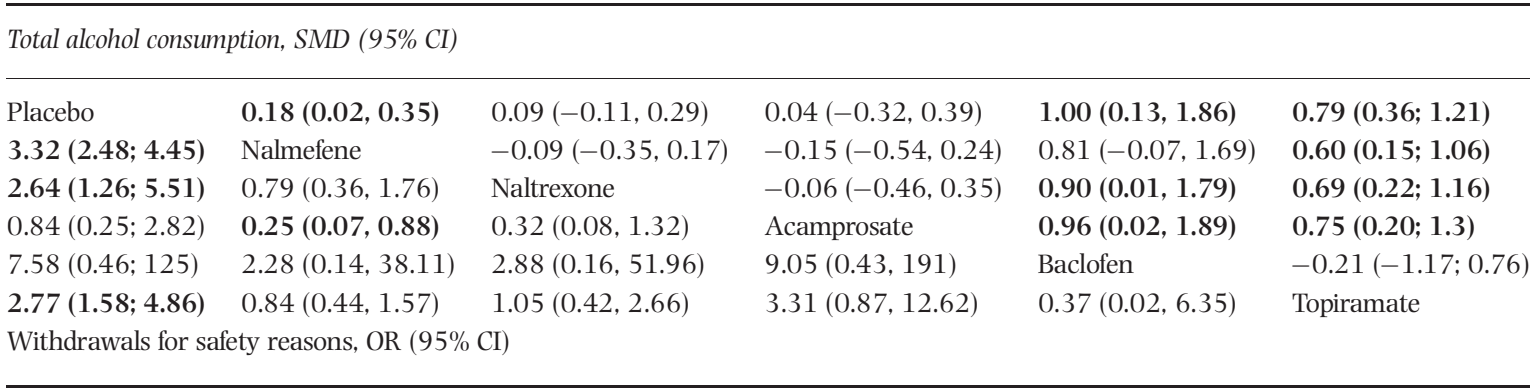

Efficacy indices between treatments (in rows) and treatment (in columns) are presented with their 95\% confidence interval (CI); for total alcohol consumption (top right corner), a negative value is in favour of the treatment in the row; for the withdrawals for safety reasons (lower-left corner), a value below 1 is in favour of the treatment in the row; bold values are statistically significant. $\mathrm{SMD}=$ standardized mean difference; $\mathrm{OR}=\mathrm{odds}$ ratio; $\mathrm{CI}=\mathrm{confidence}$ interval.

a

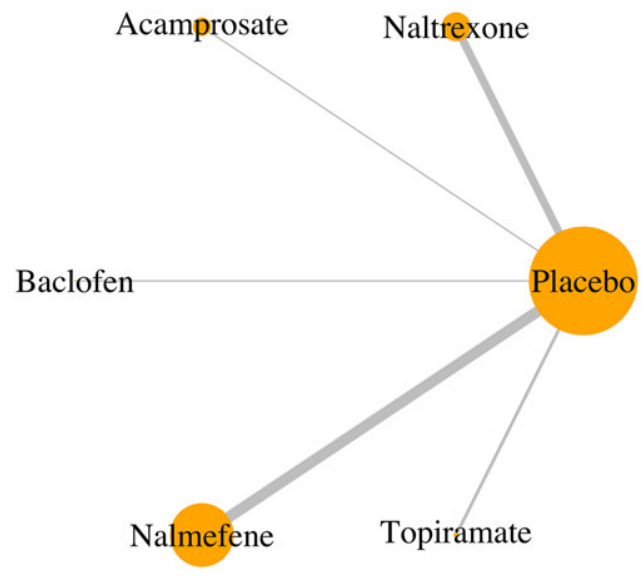

$\mathrm{b}$



Figure 3 Network geometry for the total alcohol consumption outcome (a) and the withdrawals for safety reasons outcome (b). Each node is proportional to the number of patients for whom the outcome is available. Each edge is proportional to the number of studies for which the comparison is available.

in an emerging field under pressure from both financial conflicts of interest (e.g. the initial intensive, targeted marketing of nalmefene) and non-financial conflicts of interest (e.g. widespread public support, in part via internet forums, for the use of baclofen).

Furthermore, our study denotes a lack of a coherent research agenda (placebo is the most widely studied intervention and no study has compared two active treatments directly) implementing consensual methods to assess and measure outcomes [63].

We performed network meta-analyses which were based completely on indirect comparisons. These analyses should not replace head-to-head RCTs, but appeared necessary as some of the treatments explored have been used offlabel for several years. Major shortcomings need to be discussed to avoid any overinterpretation of the results. First, indirect comparisons rely on a similarity assumption (their validity depends on the distribution of treatment effect modifiers) [64], and systematic differences throughout studies might bias the results. For instance, we observed that study durations were generally longer for nalmefene trials than for the other drugs. Therefore, these indirect comparisons are supported in part by the assumption of a constant effect of the treatment over time. It is noticeable that the same hypothesis is made when a surrogate outcome is measured in the short or medium term to deduce the long-term effect of pharmacologically controlled drinking on health outcomes. Secondly, we found considerable heterogeneity (especially for naltrexone and topiramate), which reduces the validity of the indirect comparisons. We identified the sex ratio as a possible explanation for this heterogeneity in explanatory analyses. In post-hoc sensitivity analyses, the results appeared robust, but heterogeneity was still present. Other sources of heterogeneity are possible, such as the great variety of psychological co-interventions used in the different trials, ranging from simple medical management to standardized interventions such as BRENDA. In addition, although we carefully selected studies including non-abstinent patients, different treatment goals across studies could explain some of this heterogeneity. It might be hypothesized that patients engaged in abstinence-orientated treatment may be likely to 


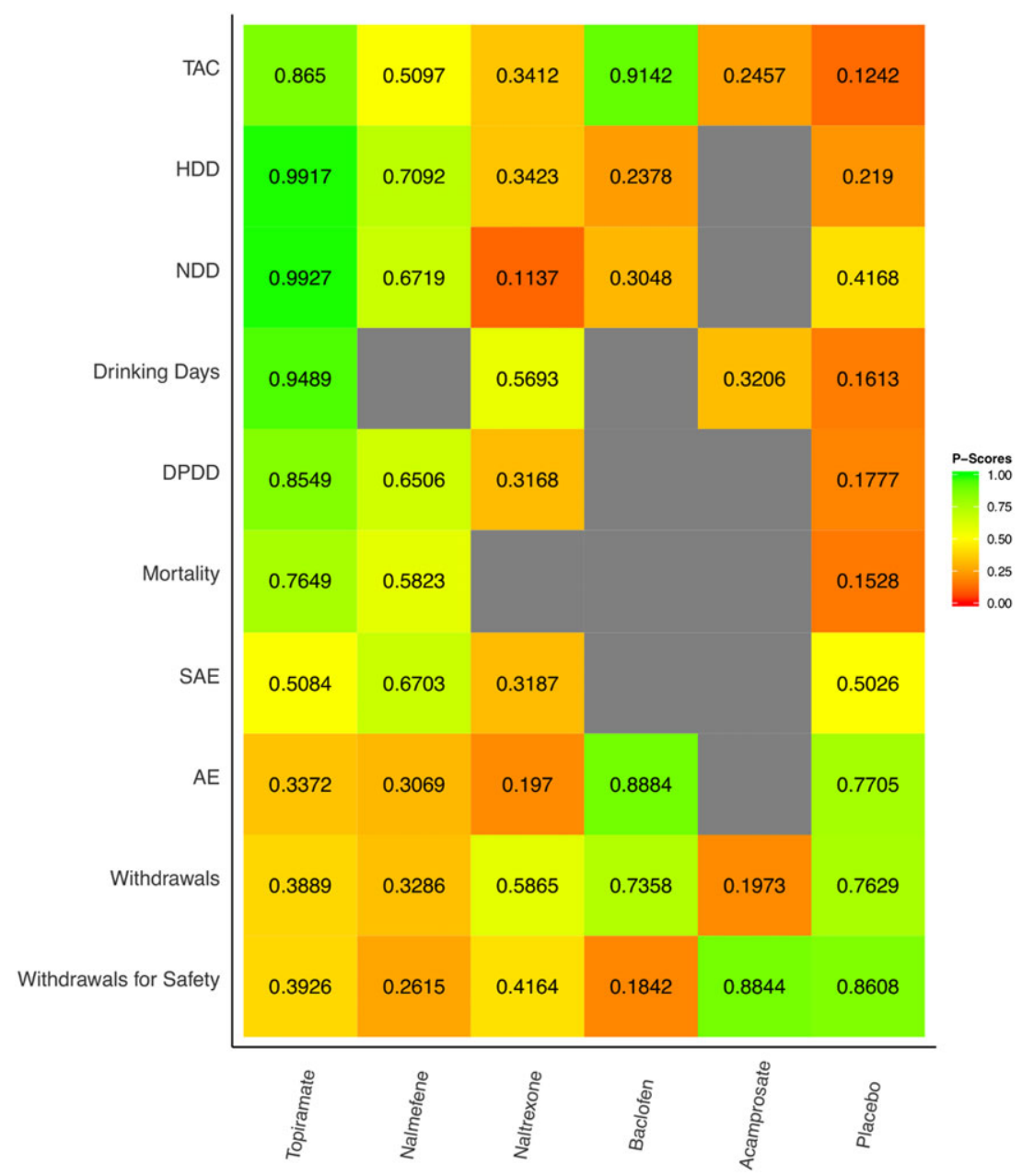

Figure 4 P-scores estimations. P-scores are values comprised between 0 and I that measure the mean extent of certainty that a treatment is better than the competing treatments. The closer to I the P-score, the better the treatment. TAC = Total alcohol consumption; HDD = Heavy drinking days; NDD = Non drinking days; DPDD = Drinks per drinking day; SAE = Serious adverse events; AE = Adverse events.

stop taking the drug when they begin drinking because they see this as treatment failure. The exact treatment goals were reported inconsistently in the different studies, and it was not possible to take this information into account.

A publication bias and a selective outcome reporting bias might also account for some of the effects we observed. Finally, because nalmefene has approval in Europe, the EMA granted us access to unpublished studies and study reports, and we included what we believe to be all available relevant data from completed studies on nalmefene that met our inclusion criteria. This was not the case for the other treatments, and this could have biased our results towards an underestimation of the comparative effectiveness of nalmefene.

While comparative effectiveness is central to drug evaluation, this evidence was lacking at the time nalmefene was approved. The main concerns relate to the lack of meaningful comparisons with naltrexone, which is a very similar compound. When the NICE (the National Institute for Health and Care Excellence) evaluated nalmefene, the pharmaceutical company Lundbeck (the compound patent holder) stated that all the naltrexone studies had limitations in the data reported, meaning that an indirect comparison could not be performed [65]. Indeed, our independent appraisal found that the naltrexone studies included were highly heterogeneous and had an unclear or high risk of selective outcome reporting bias, compromising a definitive answer to the question of comparative effectiveness between the two compounds. Direct comparisons are therefore needed.

In disagreement with the above statement, reported to NICE, a Lundbeck-sponsored indirect comparison of nalmefene and naltrexone was published recently and found a superiority of nalmefene [17]. Inclusion criteria in this meta-analysis were close to ours, except that we were more stringent concerning the duration of the abstinence period allowed before entering the trial. However, this study compared subgroup analyses on nalmefene RCTs with naltrexone RCTs as a whole. The validity of these a posteriori-defined subgroup analyses has been criticized 
$[6,66]$, and in the context of an indirect comparison it challenges the similarity assumption dramatically. While we did not find any significant difference between naltrexone and placebo, indirect comparisons showed no evidence of a superiority of nalmefene over naltrexone. In addition, both drugs were linked to more adverse events than placebo.

\section{Perspectives}

As shown previously, nalmefene has small effects on surrogate outcomes (with moderate confidence in the evidence) and no demonstrated effect on health outcomes [8]. No evidence was found of superiority against another drug. Among the currently ongoing registered postmarketing studies, none is to address these issues (i.e. lack of power for health outcomes and/or lack of comparisons) [7].

No efficacy was found here for either naltrexone or acamprosate. Regarding baclofen, we found very poor evidence for its use (very low confidence in evidence that was very inconsistent). We found only small studies involving low doses (ranging from 30 to $50 \mathrm{mg} /$ day). These doses were well below the doses that can be prescribed in dayto-day practice. Indeed, the prescription of high doses (up to $300 \mathrm{mg}$ /day) has increased rapidly among alcohol specialists [67], while the marketing authorization for spasticity forbids the use of more than $40 \mathrm{mg} /$ day and warns against associated alcohol use [68]. These practices were based initially on case reports, case series or rudimentary cohort studies [69]. A 1-year efficacy clinical trial in this specific indication was completed in France (BACLOVILLE study, NCT01604330) and was partially presented at a congress. Only the results concerning mortality and the primary outcome were released. They suggested efficacy on consumption outcomes, with an absolute difference in likelihood of success (defined as abstinence or a low level of consumption) of $20.3 \%$ (95\% CI $=7.3,33.3)$ in favour of baclofen. No effect was found on mortality with, respectively, seven of 162 deaths in the baclofen group (five due to alcohol) and three of 158 in the placebo group (one due to alcohol). Although not significant (the study was powered to evidence benefit on consumption outcomes, and not on mortality) this result suggests that the effect observed on consumption outcomes does not necessarily reflect efficacy on health outcomes. As in other studies, attrition was high (32\% lost to follow-up). Data on other outcomes, including the outcomes relevant for our meta-analysis, have still been not released, despite a primary completion date in September 2014 and a database lock in October 2015. It is estimated that tens of thousands of patients with alcohol use disorders are targeted [70] in France by a very specific supervision procedure ('temporary recommendation for use') [68]. Therefore, the complete results, enabling the possible benefits to be weighed against the known safety issues [71], especially in the case of deliberate acute baclofen poisoning [72], are required urgently [73].

Interestingly, despite the major limitations we have raised, our data suggests that topiramate could be considered as the most effective treatment, with medium to large effect sizes on most consumption outcomes (but with very low confidence in the evidence). This result, which should be considered exploratory, is nonetheless interesting to analyse. Indeed, it would be tempting to promote the use of topiramate in alcohol dependence and AUDs, as it is associated with a significant decrease in alcohol consumption and its safety profile does not appear to differ from that of placebo for adverse events, serious adverse events or mortality. Previous evidence does not support this idea, however, as the use of topiramate is known to result, for example, in negative cognitive side effects such as deterioration in verbal fluency, language comprehension, working memory and visual block tapping [74]. The absence of difference between topiramate and the other drugs could be explained by insufficient study duration, insufficient power and/or by poor quality in the reporting of harmful effects or events [75]. In our opinion, this example illustrates the fact that surrogate outcomes should not be used alone. We feel that the EMA should revise their standards and support the implementation of adequately designed trials on health outcomes. In any event, there is an urgent need to provide policymakers with evidence as to which of these pharmacologically controlled drinking approaches can be translated effectively into a real harm reduction strategy.

To conclude, our results suggest that no treatment currently has high-grade evidence for pharmacologically controlled drinking in the treatment of patients suffering from alcohol dependence or alcohol use disorders. At best, some showed low to medium efficacy in reducing drinking, but across a range of studies with a high risk of bias. Although based on all available data in the public domain, this metaanalysis found no evidence of any benefit of the use of drugs aiming for a controlled drinking strategy on health outcomes. We invite researchers and stakeholders to set up a coherent agenda to demonstrate that pharmacologically controlled drinking can be translated into genuine harm reduction for patients. From the clinical perspective, while this new approach is often presented as a 'paradigm shift' in terms of therapeutics, doctors and patients should be informed that the critical examination of the pros and cons of the evidence clearly questions the current guidelines that promote drugs in this indication.

\section{Declaration of interests}

None. All authors completed the Unified Competing Interest form at http://www.icmje.org/coi_disclosure.pdf (available on request from the corresponding author) and declare that C.P. was a trainee in Servier (pharmacokinetics 
department) for 6 months in 2013; R.D., A.H., C.L., R.B. and B.L. have had no relationships with any company that might have an interest in the submitted work in the previous 3 years; F.N. has had relationships (travel/accommodation expenses covered/reimbursed) with Servier, BMS, Lundbeck and Janssen, who might have an interest in the work submitted in the previous 3 years.

\section{Acknowledgements}

We would like to thank Dr Jonas, who shared the bibliographic database of a previous systematic review, all the authors who agreed to provide additional information concerning their studies, Dr Rücker for the information she provided regarding the use of the netmeta library, Angela Swaine Verdier for revising the English and Karima Hammas for her help in formatting the manuscript. This study was funded by Rennes CHU (CORECT : Comité de la Recherche Clinique et Translationnelle).

\section{References}

1. Klingemann J. Acceptance of reduced-risk drinking as a therapeutic goal within the polish alcohol treatment system. Alcohol Alcohol 2016; 51: 436-41.

2. Rosenberg H., Davis A. K. Differences in the acceptability of non-abstinence goals by type of drug among American substance abuse clinicians. J Subst Abuse Treat 2014; 46: 214-8.

3. European Medicines Agency. Assessment report: Selincrointernational non-proprietory name: nalmefene. EMA/ 78844/2013. Dec 13, 2012. Available at: http://www.ema. europa.eu/docs/en_GB/document_library/EPAR_-_Public_as sessment_report/human/002583/WC500140326.pdf (accessed 22 Aug 2016) (Archived at http://www.webcitation.org/ 6sw9M3XxC).

4. Braillon A. Nalmefene in alcohol misuse: junk evaluation by the European medicines agency. BMJ 2014; 348: g2017.

5. Spence D. Bad medicine: nalmefene in alcohol misuse. BMJ 2014; 348: g1531.

6. Fitzgerald N., Angus K., Elders A., de Andrade M., Raistrick D., Heather N. et al. Weak evidence on nalmefene creates dilemmas for clinicians and poses questions for regulators and researchers. Addiction 2016; 111: 1477-87.

7. Naudet F., Palpacuer C., Boussageon R., Laviolle B. Evaluation in alcohol use disorders-insights from the nalmefene experience. BMC Med 2016; 14: 119.

8. Palpacuer C., Laviolle B., Boussageon R., Reymann J. M., Bellissant E., Naudet F. Risks and benefits of nalmefene in the treatment of adult alcohol dependence: a systematic literature review and meta-analysis of published and unpublished double-blind randomized controlled trials. PLOS Med 2015; 12: e1001924.

9. Stevenson M., Pandor A., Stevens J. W., Rawdin A., Rice P., Thompson J. et al. Nalmefene for reducing alcohol consumption in people with alcohol dependence: an evidence review group perspective of a NICE single technology appraisal. PharmacoEconomics 2015; 33: 833-47.

10. Garbutt J. C. Efficacy and tolerability of naltrexone in the management of alcohol dependence. Curr Pharm Des 2010; 16: 2091-7.
11. Teng Goh E., Morgan M. Y. Pharmacotherapy for alcohol dependence: the why, the what and the wherefore. Aliment Pharmacol Ther 2017; 45: 865-82.

12. Rolland B., Paille F., Gillet C., Rigaud A., Moirand R., Dano C. et al. Pharmacotherapy for alcohol dependence: the 2015 recommendations of the French alcohol society, issued in partnership with the European Federation of Addiction Societies. CNS Neurosci Ther; 2016: 25-37.

13. Soyka M., Kranzler H. R., Hesselbrock V., Kasper S., Mutschler J., Moller H. J. Guidelines for biological treatment of substance use and related disorders, part 1: Alcoholism, first revision. World J Biol Psychiatry 2017; 18: 86-119.

14. Burton R., Henn C., Lavoie D., O'Connor R., Perkins C., Sweeney K. et al. A rapid evidence review of the effectiveness and cost-effectiveness of alcohol control policies: an English perspective. Lancet 2017; 389: 1558-80.

15. Hall W., Zador D. The alcohol withdrawal syndrome. Lancet 1997; 349: 1897-900.

16. Jonas D. E., Amick H. R., Feltner C., Bobashev G., Thomas K., Wines R. et al. Pharmacotherapy for adults with alcohol use disorders in outpatient settings: a systematic review and meta-analysis. JAMA 2014; 311: 1889-900.

17. Soyka M., Friede M., Schnitker J. Comparing Nalmefene and naltrexone in alcohol dependence: are there any differences? Results from an indirect meta-analysis. Pharmacopsychiatry 2016; 49: 66-75.

18. Higgins J. P., Altman D. G., Gotzsche P. C., Juni P., Moher D., Oxman A. D. et al. The Cochrane Collaboration's tool for assessing risk of bias in randomised trials. BMJ 2011; 343: d5928.

19. Higgins J. P. T., Green S., editors. Cochrane handbook for systematic reviews of interventions. Cochrane Collaboration. Version 5.1.0. Available at: http://handbook-5-1.cochrane. org/ (accessed 23 August 2017) (Archived at http://www. webcitation.org/6sw9vRlcB).

20. R Development Core Team. R: a Language and Environment for Statistical Computing. Vienna: R Foundation for Statistical Computing; 2009.

21. Schwarzer G. Meta: general package for meta-analysis, version 3.6-0. 27 May 2014. Available at: https://cran.r-project.org/web/packages/meta/ (accessed 23 August 2017) (Archived at http://www.webcitation.org/6swAAXySQ).

22. Rücker G., Schwarzer G., Krahn U., König J. netmeta: Network meta-analysis using frequentist methods. R package version 0.8-0, 2015. (Available at: https://cran.r-project. org/web/packages/netmeta/ (accessed 23 August 2017) (Archived at http://www.webcitation.org/6swALYuOx).

23. Viechtbauer $\mathrm{W}$. Conducting meta-analyses in $\mathrm{R}$ with the metafor package. J Stat Softw 2010; 36: 1-48. Available at: http://www.jstatsoft.org/v36/i03/ (accessed 26 August 2016) (Archived at http://www.webcitation.org/6swAYx7cG).

24. Liberati A., Altman D. G., Tetzlaff J., Mulrow C., Gotzsche P. C., Ioannidis J. P. et al. The PRISMA statement for reporting systematic reviews and meta-analyses of studies that evaluate health care interventions: explanation and elaboration. J Clin Epidemiol 2009; 62: e1-34.

25. Hutton B., Salanti G., Caldwell D. M., Chaimani A., Schmid C. H., Cameron C. et al. The PRISMA extension statement for reporting of systematic reviews incorporating network meta-analyses of health care interventions: checklist and explanations. Ann Intern Med 2015; 162: 777-84.

26. Yusuf S., Peto R., Lewis J., Collins R., Sleight P. Beta blockade during and after myocardial infarction: an overview of the randomized trials. Prog Cardiovasc Dis 1985; 27: 335-71. 
27. Rucker G. Network meta-analysis, electrical networks and graph theory. Res Synth Meth 2012; 3: 312-24.

28. Rucker G., Schwarzer G. Ranking treatments in frequentist network meta-analysis works without resampling methods. BMC Med Res Methodol 2015; 15: 58.

29. Sterne J. A., Sutton A. J., Ioannidis J. P., Terrin N., Jones D. R., Lau J. et al. Recommendations for examining and interpreting funnel plot asymmetry in meta-analyses of randomised controlled trials. BMJ 2011; 343: d4002.

30. Salanti G., Del Giovane C., Chaimani A., Caldwell D. M., Higgins J. P. Evaluating the quality of evidence from a network meta-analysis. PLOS ONE 2014; 9: e99682.

31. Anton R. F., Pettinati H., Zweben A., Kranzler H. R., Johnson B., Bohn M. J. et al. A multi-site dose ranging study of nalmefene in the treatment of alcohol dependence. J Clin Psychopharmacol 2004; 24: 421-8.

32. Gual A., He Y., Torup L., van den Brink W., Mann K. A randomised, double-blind, placebo-controlled, efficacy study of nalmefene, as-needed use, in patients with alcohol dependence. Eur Neuropsychopharmacol 2013; 23 : $1432-42$.

33. Karhuvaara S., Simojoki K., Virta A., Rosberg M., Loyttyniemi E., Nurminen T. et al. Targeted nalmefene with simple medical management in the treatment of heavy drinkers: a randomized double-blind placebo-controlled multicenter study. Alcohol Clin Exp Res 2007; 31: 1179-87.

34. Mann K., Bladstrom A., Torup L., Gual A., van den Brink W. Extending the treatment options in alcohol dependence: a randomized controlled study of as-needed nalmefene. Biol Psychiatry 2013; 73: 706-13.

35. Mason B. J., Ritvo E. C., Morgan R. O., Salvato F. R., Goldberg G., Welch B. et al. A double-blind, placebo-controlled pilot study to evaluate the efficacy and safety of oral nalmefene $\mathrm{HCl}$ for alcohol dependence. Alcohol Clin Exp Res 1994; 18: 1162-7.

36. Mason B. J., Salvato F. R., Williams L. D., Ritvo E. C., Cutler R. B. A double-blind, placebo-controlled study of oral nalmefene for alcohol dependence. Arch Gen Psychiatry 1999; 56: 719-24.

37. van den Brink W., Sorensen P., Torup L., Mann K., Gual A. Long-term efficacy, tolerability and safety of nalmefene asneeded in patients with alcohol dependence: a 1-year, randomised controlled study. J Psychopharmacol 2014; 28 : 733-44.

38. Ahmadi J., Babaeebeigi M., Maany I., Porter J., Mohagheghzadeh M., Ahmadi N. et al. Naltrexone for alcohol-dependent patients. Ir J Med Sci 2004; 173: 34-37.

39. Anton R. F., Myrick H., Wright T. M., Latham P. K., Baros A. M., Waid L. R. et al. Gabapentin combined with naltrexone for the treatment of alcohol dependence. Am J Psychiatry 2011; 168: 709-17.

40. Anton R., Baros A., Latham P., Randall P., Stewart S., Vergne D. et al. Naltrexone plus aripiprazole compared to naltrexone alone and placebo in the treatment of alcohol dependencea double blind pilot study. Neuropsychopharmacology 2011; 36: S234-5.

41. Davidson D., Saha C., Scifres S., Fyffe J., O'Connor S., Selzer C. Naltrexone and brief counseling to reduce heavy drinking in hazardous drinkers. Addict Behav 2004; 29: 1253-8.

42. Heinala P., Alho H., Kiianmaa K., Lonnqvist J., Kuoppasalmi K., Sinclair J. D. Targeted use of naltrexone without prior detoxification in the treatment of alcohol dependence: a factorial double-blind, placebo-controlled trial. J Clin Psychopharmacol 2001; 21: 287-92.
43. Killeen T. K., Brady K. T., Gold P. B., Simpson K. N., Faldowski R. A., Tyson C. et al. Effectiveness of naltrexone in a community treatment program. Alcohol Clin Exp Res 2004; 28: 1710-7.

44. Kranzler H. R., Modesto-Lowe V., Kirk J. Naltrexone vs. nefazodone for treatment of alcohol dependence. A placebo-controlled trial. Neuropsychopharmacology 2000; 22: 493-503.

45. Kranzler H. R., Armeli S., Tennen H., Blomqvist O., Oncken C., Petry N. et al. Targeted naltrexone for early problem drinkers. J Clin Psychopharmacol 2003; 23: 294-304.

46. Kranzler H. R., Tennen H., Armeli S., Chan G., Covault J., Arias A. et al. Targeted naltrexone for problem drinkers. J Clin Psychopharmacol 2009; 29: 350-7.

47. Morgenstern J., Kuerbis A. N., Chen A. C., Kahler C. W., Bux D. A. Jr., Kranzler H. R. A randomized clinical trial of naltrexone and behavioral therapy for problem drinking men who have sex with men. J Consult Clin Psychol 2012; 80: $863-75$.

48. Morris P. L., Hopwood M., Whelan G., Gardiner J., Drummond E. Naltrexone for alcohol dependence: a randomized controlled trial. Addiction 2001; 96: 1565-73.

49. O'Malley S. S., Corbin W. R., Leeman R. F., DeMartini K. S., Fucito L. M., Ikomi J. et al. Reduction of alcohol drinking in young adults by naltrexone: a double-blind, placebocontrolled, randomized clinical trial of efficacy and safety. $J$ Clin Psychiatry 2015; 76: e207-13.

50. Oslin D. W., Lynch K. G., Pettinati H. M., Kampman K. M., Gariti P., Gelfand L. et al. A placebo-controlled randomized clinical trial of naltrexone in the context of different levels of psychosocial intervention. Alcohol Clin Exp Res 2008; 32: 1299-308.

51. Tidey J. W., Monti P. M., Rohsenow D. J., Gwaltney C. J., Miranda R. Jr., McGeary J. E. et al. Moderators of naltrexone's effects on drinking, urge, and alcohol effects in nontreatment-seeking heavy drinkers in the natural environment. Alcohol Clin Exp Res 2008; 32: 58-66.

52. Mason B. J., Goodman A. M., Chabac S., Lehert P. Effect of oral acamprosate on abstinence in patients with alcohol dependence in a double-blind, placebo-controlled trial: the role of patient motivation. J Psychiatr Res 2006; 40: 383-93.

53. Addolorato G., Caputo F., Capristo E., Domenicali M., Bernardi M., Janiri L. et al. Baclofen efficacy in reducing alcohol craving and intake: a preliminary double-blind randomized controlled study. Alcohol Alcohol 2002; 37: 504-8.

54. Addolorato G., Leggio L., Ferrulli A., Cardone S., Bedogni G., Caputo F. et al. Dose-response effect of baclofen in reducing daily alcohol intake in alcohol dependence: secondary analysis of a randomized, double-blind, placebo-controlled trial. Alcohol Alcohol 2011; 46: 312-7.

55. Garbutt J. C., Kampov-Polevoy A. B., Gallop R., Kalka-Juhl L., Flannery B. A. Efficacy and safety of baclofen for alcohol dependence: a randomized, double-blind, placebo-controlled trial. Alcohol Clin Exp Res 2010; 34: 1849-57.

56. Ponizovsky A. M., Rosca P., Aronovich E., Weizman A., Grinshpoon A. Baclofen as add-on to standard psychosocial treatment for alcohol dependence: a randomized, doubleblind, placebo-controlled trial with 1 year follow-up. J Subst Abuse Treat 2015; 52: 24-30.

57. Johnson B. A., Ait-Daoud N., Bowden C. L., DiClemente C. C., Roache J. D., Lawson K. et al. Oral topiramate for treatment of alcohol dependence: a randomised controlled trial. Lancet 2003; 361: 1677-85. 
58. Johnson B. A., Rosenthal N., Capece J. A., Wiegand F., Mao L., Beyers K. et al. Topiramate for treating alcohol dependence: a randomized controlled trial. JAMA 2007; 298: 1641-51.

59. Knapp C. M., Ciraulo D. A., Sarid-Segal O., Richardson M. A., Devine E., Streeter C. C. et al. Zonisamide, topiramate, and levetiracetam efficacy and neuropsychological effects in alcohol use disorders. J Clin Psychopharmacol 2015; 35: $34-42$.

60. Kranzler H. R., Covault J., Feinn R., Armeli S., Tennen H., Arias A. J. et al. Topiramate treatment for heavy drinkers: moderation by a GRIK1 polymorphism. Am J Psychiatry 2014; 171: 445-52.

61. Addolorato G., Leggio L. Safety and efficacy of baclofen in the treatment of alcohol-dependent patients. Curr Pharm Des 2010; 16: 2113-7.

62. Mills E. J., Thorlund K., Ioannidis J. P. Demystifying trial networks and network meta-analysis. BMJ 2013; 346: f2914.

63. Gargon E., Williamson P. R., Altman D. G., Blazeby J. M., Tunis S., Clarke M. The COMET initiative database: progress and activities update (2015). Trials 2017; 18: 54.

64. Jansen J. P., Naci H. Is network meta-analysis as valid as standard pairwise meta-analysis? It all depends on the distribution of effect modifiers. BMC Med 2013; 11: 159.

65. National Institute for Health and Care Excellence. Nalmefene for reducing alcohol consumption in people with alcohol dependence: evaluation report. 2014. Available at: https:// www.nice.org.uk/guidance/ta325?unlid=3629887952016 628192516 (accessed 24 August 2016) (Archived at http:// www.webcitation.org/6swAnhc4N).

66. Naudet F. Comparing nalmefene and naltrexone in alcohol dependence: is there a spin? Pharmacopsychiatry 2016; 49: 260-1.

67. Rolland B., Paille F., Fleury B., Cottencin O., Benyamina A., Aubin H. J. Off-label baclofen prescribing practices among French alcohol specialists: results of a national online survey. PLOS ONE 2014; 9: e98062.

68. Braillon A. Recommendations of French alcohol society and European Federation of Addiction Societies. CNS Neurosci Ther 2016; 22: 535-6.

69. Rolland B., Auffret M., Franchitto N. Safety reports on the offlabel use of baclofen for alcohol-dependence: recommendations to improve causality assessment. Expert Opin Drug Saf 2016; 15: 747-51.

70. Degrassat-Theas A., Bocquet F., Sinegre M., Peigne J., Paube $\mathrm{P}$. The 'temporary recommendations for use': a dual-purpose regulatory framework for off-label drug use in France. Health Policy 2015; 119: 1399-405.

71. Harm Reduction International. What is harm reduction? A position statement from harm reduction international. 2016. Available at: https://www.hri.global/what-isharm-reduction (accessed 23 August 2017) (Archived at http://www.webcitation.org/6swBVmgy4).

72. Pommier P., Debaty G., Bartoli M., Viglino D., Carpentier F., Danel V. et al. Severity of deliberate acute baclofen poisoning: a nonconcurrent cohort study. Basic Clin Pharmacol Toxicol 2014; 114: 360-4.

73. Braillon A., Naudet F. Baclofen and alcohol use disorders: from miracle to mirage. Eur Neuropsychopharmacol 2017; 27: 691-2.

74. Fritz N., Glogau S., Hoffmann J., Rademacher M., Elger C. E., Helmstaedter C. Efficacy and cognitive side effects of tiagabine and topiramate in patients with epilepsy. Epilepsy Behav 2005; 6: 373-81.

75. Ioannidis J. P., Contopoulos-Ioannidis D. G. Reporting of safety data from randomised trials. Lancet 1998; 352: 1752-3.

\section{Supporting Information}

Additional Supporting Information may be found online in the supporting information tab for this article.

Appendix S1 Recommended doses for each treatment in adults.

Appendix S2 List of outcomes reported for each study.

Appendix S3 Results of direct comparisons against placebo. Appendix S4 Results of indirect comparisons: network plots.

Appendix S5 Results of indirect comparisons: contrast tables.

Appendix S6 Evaluation of the quality of evidence using Grading of Recommendations Assessment, Development and Evaluation (GRADE) framework.

Appendix S7 Preferred Reporting Items for Systematic Reviews and Meta-Analyses (PRISMA) Checklist.

Appendix S8 Protocol. 